\title{
Clima organizacional en un hospital público de Quindío Colombia
}

\author{
Rubén Darío Agudelo Loaiza, Claudia Echeverry Ríos, Claudia Lorena Echeverry Moreno, \\ William Beltrán Cárdenas, Marco Antonio Moreno
}

Facultad de Ciencias de la Salud, Universidad Católica de Manizales, Manizales, Caldas, Colombia.

\begin{abstract}
Resumen: Objetivo: Caracterizar el clima organizacional percibido por funcionarios asistenciales y administrativos en un hospital publico de Quindio-Colombia en 2015. Método: Estudio cuantitativo de tipo correlacional, que mide el clima organizacional de un hospital de baja complejidad mediante la caracterización de doce dimensiones. La población estudio la conformaron 114 funcionarios asistenciales y administrativos pertenecientes a un hospital público de Quindío-Colombia. Resultados: La medición del clima organizacional dio como resultado un promedio global de 69,81, con una calificación de 3,89 catalogándose como un nivel medio para el clima organizacional. Se evidenció una relación positiva altamente significativa entre el clima organizacional con la dimensión de relaciones interpersonales y de esta última con la coordinación externa. Conclusión: Se evidencia la importancia que para el funcionario tiene, desde su punto de vista, la "interacción amigable" que se interpreta como la relación respetuosa y efectiva con sus compañeros de área, necesaria para un ambiente de trabajo en equipo; de igual manera se evidenció la importancia de tener en cuenta las relaciones interpersonales con la coordinación externa lo que se puede traducir como el valor que el funcionario le confiere al liderazgo participativo en la percepción del clima organizacional.
\end{abstract}

Palabras clave: Cultura Organizacional, Instituciones de Salud, Recursos Humanos en Salud.

\section{Clima organizacional em um hospital público de Quindío Colombia}

Resumo: Objetivo: Caracterizar o clima organizacional percebido por funcionários assistenciais e administrativos em um hospital público do departamento de Quindío-Colômbia, em 2015. Método: Estudo quantitativo de tipo correlacional, o qual mede o clima organizacional de um hospital de baixa complexidade através da caracterização de doze dimensões. A população estudada foi conformada por 114 funcionários assistenciais e administrativos pertencentes a um hospital público do departamento de Quindío na Colômbia. Resultados: A medição do clima organizacional apresentou como resultado uma média global de 69,81, com uma qualificação de 3,89 catalogando-se como um nível médio para o clima organizacional. Evidenciou-se uma relação positiva altamente significativa entre o clima organizacional com a dimensão das relações interpessoais e desta última com a coordenação externa. Conclusão: Evidencia-se a importância que tem para o funcionário, desde sua perspectiva, a "interação amigável" a qual se interpreta como a relação respeitosa e efetiva com seus colegas de área, necessário para um ambiente de trabalho em equipe. Do mesmo modo, evidenciou-se a importância d as relações interpessoais com a coordenação externa, o qual pode se traduzir como o valor que o funcionário lhe dá à liderança participativa na percepção do clima organizacional.

Palavras-chave: Cultura Organizacional, Instalações de Saúde, Recursos Humanos em Saúde.

Autor para la correspondencia: Rubén Darío Agudelo Loaiza, Programa Especialización en Administración de la Salud, Facultad de Ciencias de la Salud, Universidad Católica de Manizales, Carrera 23 No.60-63, Manizales, Caldas, Colombia, e-mail: ragudelo@ucm.edu.co

Recibido Ago. 29, 2016; $1^{\text {a }}$ Revisión Dic. 5, 2016; 2 Revisión Mayo. 2, 2017; Aceptado Jun. 2, 2017. 


\title{
Organizational climate in a public hospital from Quindio Colombia
}

\begin{abstract}
Objective: To characterize the organizational climate perceived by the assistance and administrative staff members at a public hospital in Quindio-Colombia 2015. Method: A quantitative correlational study, which measures the organizational climate of a low complexity hospital through the characterization of twelve dimensions. The population being studied was conformed by 114 assistance and administrative staff members who belong to a public hospital in Quindío-Colombia. Results: The measurement of the organizational climate provided a result of a global average of 69.81 with a score of 3.9 being classified as a medium level for the organizational climate. There was evidence of a highly meaningful positive bond between the organizational climate and the dimension of interpersonal relationships and between this one with the external coordination. Conclusion: There is evidence of the importance given by the staff members to the "friendly interaction" that is interpreted as a respectful and effective relationship with their co-workers, which is, in turn, necessary for the generation of a teamwork environment. Additionally, it was found that a high degree of importance regarding interpersonal relationships with the external coordination is given; fact that can be understood as the value staff members confere to the participative leadership within their perception of organizational climate.
\end{abstract}

Keywords: Organizational Culture, Health Care Institutions, Human Resources in Health.

\section{Introducción}

Las dinámicas organizacionales que se viven actualmente, hacen que las personas se sometan constantemente a situaciones cambiantes e interacciones diarias que interfieren directamente en la productividad y el logro de los objetivos institucionales, por las diversas situaciones vividas en las relaciones interpersonales, la comunicación, calidad del trabajo, toma de decisiones, horizonte institucional, procesos de cambio y tipo de liderazgo (SALCEDO; ROMERO, 2006).

Autores como Lewin, consideran que el clima organizacional es una concepción útil para enlazar los aspectos objetivos de la organización (FLORES, 2014). Otros autores como Méndez (2006) han propuesto diversos planteamientos acerca del significado de clima organizacional. Pero la mayoría está de acuerdo con la estrecha relación que tiene el comportamiento del hombre con el entorno de su organización (GARCÍA SOLARTE, 2009).

Independientemente de la definición que se acepte como la más pertinente para su uso académico y/o práctico, se desea enfatizar en este trabajo realizado en nuestro medio, que el clima laboral puede ser cuantificado y gerenciado, constituyéndose este último aspecto, en un factor determinante en procesos de cambio promovidos por la gerencia, que son necesarios para que la organización se adapte de manera rutinaria a nuevos elementos internos y externos que presionan la estabilidad y crecimiento organizacional (SERLIN, 2010).
Se plantea que uno de los aspectos más importante a tener en cuenta por un gerente, es el hecho de entender cómo el cliente interno cuenta con un trato equitativo, siendo interlocutor de una comunicación honesta con sus superiores jerárquicos a través de la cual sea informado sobre las expectativas y responsabilidades, así como también de sus reconocimientos por los esfuerzos y logros obtenidos (SEGREDO PÉREZ, 2013). En términos más sencillos se propone "[...] como una necesidad de brindar un trato a las personas en la organización exactamente igual a como se quiere que ellos traten a sus clientes" (PÉREZ DE MALDONADO; MALDONADO PÉREZ; BUSTAMANTE UZCÁTEGUI , 2006, p. 231). En otras palabras: para exigir desde la gerencia, un trato óptimo a los pacientes y/o clientes externos, se requiere que el cliente interno sea tratado con la misma consideración.

Se entiende entonces que el gerenciar el clima laboral es fundamental, dado el impacto en el cumplimiento cabal de la misión (SALAZAR ESTRADA et al., 2009). Esto ratifica la necesidad de que las prácticas laborales estén dirigidas a crear un entorno afectivo que facilite los procesos de desarrollo del personal de las organizaciones, pues cualquier proyecto que desestime la influencia de las conductas afectivas del individuo en su actividad diaria, minimiza las ganancias que la organización (PÉREZ DE MALDONADO; MALDONADO PÉREZ; BUSTAMANTE UZCÁTEGUI, 2006, p. 231). 
Una de las principales funciones de la gerencia es, la creación de un clima organizacional favorable, tanto físico como mental, que induzca a las personas a contribuir espontánea y voluntariamente con sus esfuerzos, a lograr cambios y obtener los objetivos planteados para lograr mejoras a largo plazo en la productividad (CARRILLO ARRIAGA, 2011).

Las instituciones prestadores de servicios de salud, se constituyen en uno de los apoyos esenciales en una sociedad prospera y productiva, por estos motivos, diferentes investigadores a nivel internacional y nacional, se han dado a la tarea de realizar estudios que soportan el clima organizacional en los sistemas de salud y en las organizaciones encargadas de prestar dichos servicios (MARÍN BETANCUR; PAZ DELGADO, 2014).

El diagnóstico del clima organizacional en el Hospital “Dr. Luis F. Nachón”. Xalapa, Veracruz (CORTÉS JIMÉNEZ, 2009). Buscó especificar las percepciones del grupo a través de las dimensiones del clima organizacional, propuestas por la organización panamericana de la salud: motivación, liderazgo, participación y reciprocidad. En esta investigación, todos los grupos refieren una percepción no satisfactoria del clima organizacional, incluyendo estas dimensiones con sus respectivas variables. Siendo el personal de enfermería el que expresa mayor nivel de insatisfacción.

El trabajo realizado sobre el clima organizacional en la gestión del cambio para el desarrollo de la organización, realiza un acercamiento conceptual del tema y exponen las diferentes dimensiones y categorías a tener en cuenta para su medición, las cuales giran en torno a los valores de la organización, las creencias, costumbres y prácticas institucionales, que permiten esclarecer las causas de la satisfacción e insatisfacción en el trabajo y su incidencia en los cambios organizacionales (SEGREDO PÉREZ, 2013).

Por otro lado, el estudio sobre clima organizacional, realizado en los colaboradores del área administrativa del hospital regional de Cobán (SIERRA, 2015), estableció que el clima laboral del área administrativa se encontraba favorable para dichos colaboradores, dado la incidencia de factores positivos encontrados, además, hallo dificultades relacionadas con la calidad y la remuneración.

En este sentido se hace necesario continuar investigando en el fenómeno del clima organizacional en la que respecta a las instituciones prestadoras de servicios de salud, por tal motivo, la intención del presente estudio consistió en caracterizar el clima organizacional percibido por los funcionarios asistenciales y administrativos de un hospital publico, con el fin de que los hallazgos encontrados, permitieran generar acciones para mitigar las diversas dificultades encontradas en los entornos laborales.

\section{Método}

Estudio cuantitativo de tipo correlacional mediante el cual se caracteriza el clima organizacional percibido por funcionarios asistenciales y administrativos pertenecientes a un hospital publico de Quindio-Colombia durante el periodo 2015

Las dimensiones abordadas fueron: la comunicación interna, el reconocimiento, las relaciones interpersonales en el trabajo, la calidad en el trabajo, la toma de decisiones, el entorno físico, los objetivos de la institución, el compromiso, la adaptación al cambio, delegación de actividades y funciones, coordinación externa y eficiencia en la productividad.

Se midieron las variables del clima organizacional considerándolo como un

[...] fenómeno multidimensional que describe la naturaleza de las percepciones que los empleados tienen de sus propias experiencias dentro de una organización (CHIANG VERA et al., 2008, p. 66).

Los participantes fueron escogidos al azar en todas las dependencias obteniendo una muestra de 114 personas quienes fueron informadas del objetivo de la encuesta y se obtuvo su consentimiento mediante el formato escogido para tal fin. La participación en la investigación se realizó de manera voluntaria y anónima, la aplicación del instrumento de recolección de datos se realizó de manera individual y sin límite de tiempo. Se trabajó con un margen de error 5\%; nivel de confianza de 95\%. La muestra estuvo conformada por personal de planta, personal vinculado por prestación de servicios y otros que se encontraban contratados a través de terceros para labores administrativas y misionales que llevaran más de 6 meses laborales dentro del hospital; excluyendo a los colaboradores llevaran menos de seis meses de labores en la institución, se encontraran en periodo vacacional o estuvieran fuera de la institución en el momento de la aplicación del instrumento. La ejecución del proyecto fue aprobada por el comité de ética de la universidad católica de Manizales. 


\subsection{Instrumento}

Para el diagnóstico del clima organizacional, se utilizó un instrumento adoptado por el Departamento de Calidad del Ministerio de Salud de Chile, validado en una experiencia anterior por Rebeco en el año 2001 (SEGREDO PÉREZ, 2013). Se trata de un cuestionario, en el cual se consideran 12 dimensiones: Comunicación interna, reconocimiento, relaciones interpersonales, calidad en el trabajo, toma decisiones trabajo, entorno físico, objetivos de la institución, compromiso, adaptación al cambio, delegación de actividades y funciones, coordinación externa, eficiencia en la productividad; conformado por 74 enunciados que dan cuenta de 12 dimensiones del clima organizacional elaborado con respuestas basadas en escala tipo Likert de cinco puntos (Muy de acuerdo $=5$, De acuerdo $=4$, No estoy seguro $=3$, En desacuerdo $=2$, Totalmente en desacuerdo $=1$ ) (SEGREDO PÉREZ, 2013).

\subsection{Análisis estadístico}

La información fue capturada en el paquete estadístico SPSS versión 21 (Statiscal Package fort he Social Sciencies, 2015) para crear la base de datos que permitió analizar la información de manera integral. Se utilizaron medidas de tendencia central: frecuencias, porcentajes, media, valor mínimo y valor máximo, para describir las características de la muestra y las variables de estudio. El coeficiente de confiabilidad del instrumento se obtuvo a través del alfa de cronbach y la curva de normalidad de los datos mediante la prueba kolmogorov-smirnov (K-S), previa construcción de índices con valor de 0 a 100, con el objetivo de identificar la pertinencia de utilizar estadística paramétrica o no paramétrica, privilegiando la utilización de (rho) de Spearman para el análisis.

\section{Resultados}

\subsection{Caracterización sociodemográfica de los participantes}

El 71,1\% de los participantes eran de sexo femenino y $28,9 \%$ de sexo masculino; la moda de la edad fue 26 años y la media de 35 años. Con respecto al tiempo que se encontraban los participantes laborado en la institución se encontró una media es de 36 meses con tiempo menor a 0 meses y mayor a 372 meses. Los tipos de contratación que predominaban fueron la prestación de servicios y tercerización, cada uno con el $42,3 \%$, el $15 \%$ restante corresponde a funcionarios de planta (nombramiento). La escolaridad arrojó que el $42,3 \%$ de las personas contaba con estudios de nivel técnico, 33\% universitario, $15,5 \%$ bachillerato, $8,2 \%$ postgrado y $1 \%$ primaria.

\subsection{Clima organizacional}

La prueba normalidad de Kolmogorov-Smirnov muestra datos con una distribución que no es normal en el cuestionario de Clima Organizacional, obteniendo una media de 69,81 $(\mathrm{DE}=18,15)$ y una cuantificación por media de 3,89 considerados medios en la escala para el efecto.

El dato mínimo del cuestionario fue de 57.97 puntos y el máximo de 85.30 puntos. En la Tabla 1 se reportan los resultados del promedio obtenido por cada dimensión del cuestionario, evidenciando que el puntaje más alto se obtuvo en la dimensión relacionada con el compromiso y el puntaje más bajo en la que se relaciona con la coordinación externa

En cuanto a las dimensiones de este instrumento se evidencian los siguientes promedios para cada una de ellas. Mostrando en los datos obtenidos una distribución no normal.

Tabla 1. Dimensiones del clima organizacional.

\begin{tabular}{lcc}
\hline \multicolumn{1}{c}{ Dimensiones } & $\overline{\mathrm{X}}$ & DE \\
\hline Comunicación Interna & 72,93 & 16,05 \\
Reconocimiento & 62,70 & 20,65 \\
Relaciones Interpersonales & 67,71 & 19,87 \\
Calidad en el Trabajo & 79,57 & 16,49 \\
Toma Decisiones Trabajo & 65,72 & 22,77 \\
Entorno Físico & 63,18 & 22,18 \\
Objetivos de la Institución & 76,89 & 20,46 \\
Compromiso & 85,30 & 15,57 \\
Adaptación al Cambio & 60,41 & 12,92 \\
Delegación de Actividades y Funciones & 67,01 & 21,43 \\
Coordinación Externa & 57,97 & 12,76 \\
Eficiencia en la Productividad & 78,35 & 78,35 \\
\hline
\end{tabular}

Fuente: Elaboración propia. 


\subsubsection{Dimensiones del clima organizacional}

\section{- Comunicación interna}

Tuvo una correlación positiva con el resultado de clima laboral. En la medida que haya una buena comunicación interna, demostrando un coeficiente de fiabilidad de 0,943 que es considerado alto (representada en los 13 ítems por un alto coeficiente de confiabilidad) aumentará la calificación del clima laboral.

\section{- Reconocimiento}

Tuvo un coeficiente de confiabilidad alto; es una de las dimensiones que mayormente se correlacionan con la medida del clima laboral y con la dimensión 12 que describe la eficiencia en la productividad. Demostrando que en la medida que el funcionario se sienta productivo y eficiente cree ser reconocido por la organización.

\section{- Relaciones interpersonales}

Se evidencia una relación positiva altamente significativa, la mayor con relación al clima laboral y con la dimensión de relaciones interpersonales, conformado por ítems que asocian el trabajo en equipo por área, la motivación por el trabajo, la interacción otros miembros del equipo y el respeto mutuo.

\section{- Calidad en el trabajo}

Se correlaciona positivamente con la dimensión de relaciones interpersonales en el trabajo. Es probable que se interprete calidad en el trabajo como calidad del ambiente laboral.

\section{- Toma decisiones trabajo}

Se correlaciona de manera menos favorable con la dimensión 12 de eficiencia en la productividad, permitiendo inferir que se mejoraría la eficiencia en un ambiente de mayor flexibilidad con posibilidad para el funcionario de asumir decisiones propias o al menos consentidas con la dirección de la organización.

\section{- Entorno físico}

Llama la atención que no obtuvo correlaciones significativas positivas ni negativas con las otras dimensiones. Se podría decir que la condición física del entorno no tuvo un peso importante a la hora de asumir una medida del clima laboral.

\section{- Objetivos de la institución}

Tuvo la media $\mathrm{X}^{-}=76,89$, que la ubica en una escala media, indicando que, en cuanto a los objetivos de la institución hay vacíos en su conocimiento definición y aceptación de estos.

\section{- Compromiso}

Obtuvo la media más alta $(\mathrm{X}=85,30)$ demostrando que los empleados del hospital sienten un alto compromiso con la institución, se sienten con la capacidad de esforzarse al máximo y un alto grado de responsabilidad en todo momento con sus actividades a pesar del tipo de contratación cuyos casos en su mayoría no generan estabilidad laboral.

\section{- Adaptación al cambio}

Esta dimensión fue una de las más bajas evidenciando cómo los empleados son un poco renuentes al cambio.

\section{- Delegación de actividades}

Se pudo observar que la delegación de funciones es poca.

\section{- Coordinación externa}

Esta dimensión tuvo la media más baja $(\mathrm{X}=57,97)$ evidenciando el escaso trabajo en equipo, comunicación con otras dependencias, conocimiento de necesidades del mismo servicio y posiblemente problemas con los conductos regulares.

\section{- Eficiencia en la productividad}

Tuvo la media $\mathrm{X}^{-}=78,35$ esta dimensión tuvo buena significación manifestando el buen uso de recursos y logro de tareas.

En cuanto a la caracterización de los objetivos se observó en las correlaciones Rho de Sperman como resulta altamente significativa las relaciones interpersonales con la comunicación interna (p 0,87), lo que nos indica que existe buen equipo de trabajo, se siente a gusto con el trabajo, hay una buena comunicación entre el equipo de trabajo.

Se pudo evidenciar que aunque esta correlación da altamente significativa, está en contra del análisis de la dimensión de comunicación que arrojo la media más baja de todos las dimensiones, pues se esperaría que las dos dieran altas. Lo que se puede observar es que esta comunicación va dirigida más que todo a la comunicación con los superiores y no con respecto al trabajo en grupo como si lo es la correlación de relaciones interpersonales.

\section{Discusión}

La percepción del clima laboral percibido por funcionarios asistenciales y administrativos que participaron en esta investigación, pertenecientes a la Empresa Social del Estado de Quindío, arrojó una calificación de 3,89 catalogándose como un nivel medio en la escala: Bajo 1-1,9; Medio 2-3,9 Alto 4-5, en contraposición a otros estudios de diagnóstico 
del clima organizacional en un hospital castrense en 2014 (RAFAEL, 2014). Donde se reveló una alta frecuencia de insatisfacción del personal en las dimensiones evaluadas; de la misma forma, un estudio realizado por Domínguez Silva, Álvarez Fuentes y Navarro Domínguez (2009), reporta que el clima organizacional de una Policlínica Universitaria puntuaba por debajo del promedio que se consideraba como límite inferior de satisfacción en todas las dimensiones analizadas. Cortés clasificó el clima organizacional global de un hospital como insatisfactorio, encontrando que solo el 9\% del personal percibía un clima satisfactorio (CORTÉS JIMÉNEZ, 2009).

En Colombia, estudios como el de Conde Martín, Figueroa Murcia y Flórez Peña (2014), abordaron la evaluación de clima laboral en una institución de salud de mayor grado de complejidad comparada con la institución de salud del presente estudio pero con la misma metodología, reportando resultados similares con una calificación para el clima de 3,4 que consideran medio o poco satisfactorio.

De acuerdo con los resultados de nuestro estudio, se puede ver que todas las dimensiones analizadas presentaron una puntuación positiva estadísticamente significativa $(\mathrm{p}=0,001)$ al relacionarlas con la comunicación interna, destacando la relación entre comunicación y relaciones interpersonales.

Aunque la bibliografía es amplia con respecto a clima laboral también es cierto que los diversos estudios a nivel internacional se caracterizan por la heterogeneidad de los planteamientos y del instrumento de medida del clima, específicamente en Colombia son escasos y como en el caso de Carmona Arias y Jaramillo Correa en 2010 en su estudio sobre el clima organizacional del hospital de Pereira (CARMONA ARIAS; JARAMILLO CORREA, 2010), enfocan su investigación en la relación negativa que constituye para el clima la falta de un modelo administrativo claramente establecido, o el de Chiang Vera, Salazar y Núñez (2007), que relaciona el clima laboral con estilos de liderazgo y riego psicosocial en empresas colombianas del sector social y de salud.

\section{Conclusion}

La dimensión de relaciones interpersonales y el clima laboral se relacionan de manera altamente significativa. Se encontró que una parte importante de los funcionarios encuestados, asocia las buenas relaciones con sus compañeros de trabajo y el respeto mutuo, como una condición importante en la percepción de "buen" clima laboral. Visto de otra manera, también se puede plantear con base en el resultado que un buen clima laboral favorece las "buenas" relaciones interpersonales y viceversa.

La correlación entre dimensiones se caracterizó por una relación directa de fuerza débil. Lo anterior se podría interpretar como la interdependencia de las dimensiones en el resultado del clima laboral lo cual implica que para impactarlo se deberían abordar todas las dimensiones analizadas, idealmente, previo un estudio más detallado por variable, diseñado específicamente o para las condiciones del Hospital objeto de estudio.

La correlación aunque positiva, más débil, se observa entre clima laboral y comunicación interna, lo cual se explicaría por una debilidad en la cultura de trabajo en equipo por un lado y por otro, que eventualmente se le da poca importancia a lo que desea la organización bien sea debido a falencias en procesos de inducción, a que no está clara la cultura organizacional y/o a que se desconocen y por lo tanto no se aplican los valores corporativos que desea reflejar la organización.

Como reflexiones a la gerencia de la organización, se propone incluir el gerenciamiento del clima laboral dentro del plan estratégico de la organización. Elaborar un programa para la gerencia del clima organizacional coordinado por personal con conocimiento en la materia, la medición del clima organizacional debe hacerse siguiendo las especificaciones científicas del caso, debe ser periódica, preferiblemente validada para el uso en instituciones de salud de bajo grado de complejidad e idealmente ejecutada por terceros.

\section{Referencias}

CARMONA ARIAS, V. M.; JARAMILLO CORREA, E. M. Estudio del clima organizacional en la ESE Hospital San Jorge de Pereira. 2010. 265 f. Trabajo de Grado (Grado en Ingeneria Industrial) - Universidad Tecnológica de Pereira, Pereira, 2010.

CARRILLO ARRIAGA, J. R. Mejoramiento del clima organizacional para disminuir accidentes y ausentismo, relacionados con estrès laboral, en Constructoras del Amm. 2011. 136 f. Tesis de Grado (Maestria en Administración de la Construción) - Universidad Autónoma de Nuevo León, San Nicolás de los Garza, 2011.

CHIANG VERA, M.; SALAZAR, C. M.; NÚÑEZ, A. Clima organizacional y satisfacción laboral en un establecimiento de salud estatal: Hospital tipo 1. Theoria. Bio Bio, Concepción, v. 16, n. 2, p. 61-76, 2007.

CHIANG VERA, M. et al. Clima organizacional y satisfacción laboral en organizaciones del sector estatal (Instituciones públicas): desarrollo, adaptación y validación 
de instrumentos. Universum, Talca, v. 23, n. 2, p. 66-85, 2008.

CONDE MARTÍN, D. M.; FIGUEROA MURCIA, C. E.; FLÓREZ PEÑA, B. Evaluación del clima organizacional de los trabajadores asistenciales del área de hospitalización del hospital Departamental San Antonio de Pitalito. 2014. 64 f. Trabajo de Grado (Especialización en administración de la salud) - Universidad Católica de Manizales, Manizales, 2014.

CORTÉS JIMÉNEZ, N. M. Diagnóstico del clima organizales. Hospital "DR. Luis F. Nachón”. Colecciones Educativas en Salud Pública, Xalapa, v. 8, p. 129-177, 2009.

DOMÍNGUEZ SILVA, S. I.; ÁLVAREZ FUENTES, F. M.; NAVARRO DOMÍNGUEZ, J. A. Clima organizacional en la Policlínica Universitaria de la familia "Carlos Verdugo". Revista Médica Electrónica, Matanzas, v. 31, n. 5, p. 1-6, 2009.

FLORES, L. Clima organizacional. Buenos Aires: Campus Pellegrini, 2014.

GARCÍA SOLARTE, M. Clima organizacional y su diagnóstico. Bogotá: Universidad de los Andes, 2009.

MARÍN BETANCUR, S. M. M.; PAZ DELGADO, A. L. Clima organizacional de la IPS Universidad Autónoma de Manizales. 2014. 132 f. Tesis (Maestria en Administracion de Negocios) - Universidad Autonoma de Manizales, Manizales, 2014.

MÉNDEZ, Á. C. E. Clima organizacional en Colombia: El IMCOC, un método de análisis para su intervención. Bogotá: Universidad del Rosario, 2006. (Colección de Lecciones de Administración).
PÉREZ DE MALDONADO, I.; MALDONADO PÉREZ, M.; BUSTAMANTE UZCÁTEGUI, S. Clima organizacional y gerencia: inductores del cambio organizacional. Investigación y Postgrado, Venezuela, v. 21, n. 2, p. 231-248, 2006.

RAFAEL, J. Diagnóstico del clima organizacional del Hospital Central de las Fuerzas Armadas de Paraguay. Memorias del Instituto de Investigaciones en Ciencias de la Salud, Asuncion, v. 12, n. 1, p. 14-25, 2014.

SALAZAR ESTRADA, J. G. et al. Clima y cultura organizacional: dos componentes esenciales en la productividad laboral. ACIMED, Ciudad de La Habana, v. 20, n. 4, p. $67-75,2009$.

SALCEDO, I.; ROMERO, J. J. Cultura organizacional y gestión de la calidad en una empresa del estado venezolano. Revista Venezolana de Gerencia, Maracaibo, v. 11, n. 33, p. 74-82, 2006.

SEGREDO PÉREZ, A. M. Clima organizacional en la gestión del cambio para el desarrollo de la organización. Revista Cubana de Salud, Ciudad de La Habana, v. 39, n. 2, p. 385-393, 2013.

SERLIN, J. Conocimiento de la gestion de las organizaciones: sistemas complejos dinamicos inestables adaptativos. 2010. 398 f. Tesis (Doctorado en Ciencias Economicas) - Universidad de Buenos Aires, Buenos Aires, 2010.

SIERRA, G. M. A. El clima laboral en los/as colaboradores/as del área administrativa del hospital regional de cobán, a.v. 2015. Trabajo de Grado (Licenciatura en Psicología Industrial/Organizacional) - Universidad Rafael Landívar, Ciudad da Guatemala, 2015.

\section{Contribución de los Autores}

Todos los autores contribuyeron igualmente en la construcción del texto. Todos los autores aproban la version final del texto. 